\title{
Transcranial Doppler Monitoring of Acute Reperfusion Therapies in Acute Isch- emic Stroke Patients with Underlying Large Vessel Occlusions
}

Odysseas Kargiotis, MD* (D); Klearchos Psychogios, MD ${ }^{*} \dagger$ (D); Apostolos Safouris, MD, (D); Georgios Magoufis, $\mathrm{MD}^{*}$ (D); Lina Palaiodimou, $\mathrm{MD}^{\dagger}$ (D); Aikaterini Theodorou, $\mathrm{MD}^{\dagger}$ (D); Eleni Bakola, $\mathrm{MD}^{\dagger, \ddagger}$ (D); Eleftherios Stamboulis, MD* (D); Christos Krogias, $\mathrm{MD}^{\mathbb{f}}$ (1); Georgios Tsivgoulis, MD, PhD, MSc, FESO, (D)

Stroke Unit, Metropolitan Hospital ${ }^{\star}$, Piraeus, Greece; Second Department of Neurology, National \& Kapodistrian University of Athens, School of Medicine, Attikon University Hospital ${ }^{\dagger}$, Athens, Greece; Department of Neurology, Thriassio General Hospital ${ }^{\ddagger}$, Elefsina, Greece; Department of Neurology, St. Josef-Hospital, Ruhr University Bochum ${ }^{0}$, Bochum, Germany; Department of Neurology, The University of Tennessee Health Science Center", Memphis, Tennessee, USA

Large vessel occlusion accounts for up to $20 \%$ of ischemic strokes. Intravenous thrombolysis and mechanical thrombectomy are two effective and complementary reperfusion therapies of acute ischemic stroke, the former exclusively applicable in patients with large vessel occlusions. Real-time, continuous monitoring of acute reperfusion therapies is only feasible with the application of transcranial Doppler (TCD) and transcranial color-coded duplex (TCCD) sonography. TCD is a bedside, easily repeatable examination that can provide with important information regarding the localization of occlusion and degree of residual flow, collateral status, real time embolization, recanalization timing and persistent target vessel patency. TCCD may also identify the presence of intracranial bleeding complicating intravenous thrombolysis or mechanical thrombectomy. TCD or TCCD monitoring of reperfusion therapies can be conducted in the emergency room, in the stroke unit and the angiography suite. In the current era of acute ischemic stroke management, TCD and TCCD do not replace other static angiographic imaging modalities but represent easily repeatable, radiation-free, bedside examinations that may disclose additional data strongly correlating with clinical outcomes which may eventually influence treatment decisions.

J Neurosonol Neuroimag 2020;12(1):10-25

Key Words: Doppler transcranial ultrasonography; Acute stroke; Tissue plasminogen activator; Thrombectomy
Received: April 28, 2020

Revised: June 11, 2020

Accepted: June 16, 2020

Correspondence:

Georgios Tsivgoulis, MD,

$\mathrm{PhD}, \mathrm{MSc}$, FESO

Second Department of Neurology, National \& Kapodistrian University of Athens, School of Medicine, Iras 39, Gerakas Attikis, Athens 15344, Greece Tel: $+30-210-583-2449$ Fax: $+30-210-583-2471$ E-mail: tsivgoulisgiorg@yahoo.gr

\section{INTRODUCTION}

Intravenous thrombolysis and mechanical thrombectomy have revolutionized acute ischemic stroke (AIS) management and consequently, have modified the natural history of the disease. ${ }^{1,2}$ The efficacy and safety of reperfusion therapies is indisputable, however, the minority of AIS patients are eventually treated $(<20 \%$ at a population-based level), primarily due to time delays, despite the efforts of different health systems to develop acute stroke treatment plans and networks. ${ }^{3,4}$
Therefore, a considerable proportion of the latest AIS studies take advantage of advanced neuroimaging in an effort to extend therapeutic time windows and increase rates of reperfusion therapies. ${ }^{5,6}$ Advanced neuroimaging is increasingly becoming readily available following the publication of the positive results from recent extended time treatment trials and the adoption of their treatment protocols by international guidelines. ${ }^{7-12}$ In any case, AIS management is continuously becoming more demanding and complicated, therefore close monitoring with continuous clinical re-assessments are 
of fundamental importance during acute reperfusion therapies. Transcranial Doppler (TCD) and transcranial color-coded duplex (TCCD) sonography represent bedside, easily repeatable, radiation-free examinations that can provide stroke physicians with important information regarding the localization of occlusion, degree of residual flow, collateral status, real time embolization, recanalization timing and persistent target vessel patency. In this review, we present the potential utility and applications of TCD and TCCD applied in the period before, during and after the acute reperfusion therapies for AIS with underlying large vessel occlusions (LVO). Most studies discussed herein have used TCD examination, particularly those performing continues monitoring. However, in comparison with TCD that it is easier to perform in emergency settings, TCCD can provide both spatial information and flow spectrum on the target vessel. Table 1 presents advantages of one modality over the other.

\section{REPERFUSION THERAPIES FOR ACUTE ISCH- EMIC STROKE}

Intravenous thrombolysis (IVT) is the only approved systemically administrated acute treatment for AIS, including LVO attributed cases. ${ }^{13}$ IVT has proven to have a substantial efficacy in reducing neurological deficit with a number needed to treat (NNT) of 4 , but also to improve 3-month disability with a NNT of 8 . The NNT for achieving excellent functional outcome ranges from 10 in patients treated within 3 hours from symptom onset and those with minor strokes, to 25 for cases with severe strokes. ${ }^{14}$ IVT is clearly a cost-effective therapy with a net benefit of $4,000 \$$ per patient receiving treatment. ${ }^{15}$

IVT is equally indicated for the treatment of AIS with and without underlying LVO. Indeed, a recent study highlights the efficacy and safety of IVT in patients with LVO or distal intracranial occlusions and mild stroke severity defined as National Institutes of Health Stroke Scale (NIHSS) scores $<6$ points. Patients receiving IVT demonstrated higher odds of functional independence and favourable functional outcome, without any significant increase in symptomatic intracranial hemorrhage (sICH) or death. Importantly, the effect of IVT was independent of preceded mechanical thrombectomy (MT). ${ }^{16}$ On the other hand, there are concerns regarding the utility of IVT in LVO patients scheduled to undergo MT. Potential disadvantages of bridging IVT with MT include increased risk of systemic or intracranial hemorrhage, orolingual angioedema, and distal embolization. ${ }^{17,18}$ However, current non-randomized data form stroke registries and meta-analyses support the use of IVT before MT since the former is associated with improved outcomes and increased recanalization rates. ${ }^{17-19}$ Randomized on-going trials intend to shed further light into the value of IVT prior to $\mathrm{MT}^{20}$

However, IVT has other important limitations: narrow time window, numerous exclusion criteria (such as prior anticoagulation treatment, recent surgery, recent history of previous AIS or myocardial infarction), avoidance of antithrombotic medications early after treatment, and low recanalization rates (up to $40 \%$ of LVOs). ${ }^{13,21}$ Concerning the latter, clot burden is a strong predictor of recanalization, and clots with $>8 \mathrm{~mm}$ length have a very low probability of dissolving (<1\%)

TABLE 1. Comparison between TCD and TCCD: advantages of one over the other

\begin{tabular}{ll}
\hline TCD & \multicolumn{1}{c}{ TCCD } \\
\hline Continuous monitoring through the placement of a head-frame & Additional spatial information \\
Higher spectral resolution & Visualization of anatomic landmarks of the brain \\
More accurate detection of microembolic signals & Trace the course of tortuous vessels \\
Most available diagnostic criteria refer to TCD studies & Easier isonation of distal braches \\
Simultaneous bilateral isonation using a head-frame & Visualization of disturbed flow (aliasing) through colour flow imaging \\
May be more efficient for patients with poor temporal windows & Potential detection of AVMs and intracranial aneurysms \\
Easier to perform at emergency settings & Detection of midline shift, hematomas through B-mode \\
Easier to transfer & Angle correction for velocity measurements
\end{tabular}

TCD; transcranial Doppler, TCCD; transcranial color-coded duplex, AVM; arteriovenous malformations. 
with rtPA infusion. ${ }^{22}$ Accordingly, the advantages of MT include more extended time windows, very few contraindications, and high recanalization rates. ${ }^{23}$ Indeed, MT compared to standard treatment leads to an absolute increase of recanalization rates by $44 \%{ }^{22}$ After the publication of several positive randomized-controlled clinical trials (RCTs), MT within 6 hours of symptom onset has become the new standard of care for LVO attributed AIS. ${ }^{12}$ Several meta-analyses have consistently documented the large clinical benefit of MT, which can be expressed as NNT of 2.6 to reduce disability by at least one level on modified Rankin score (mRS), and 6 to achieve functional independence (mRS-scores of $\mathrm{O}-2$ ), without significant increase in sICH. ${ }^{23,24}$ Finally, recent data indicates that MT coupled with best medical treatment (BMT) was associated with a significantly lower risk for 3-month mortality compared with BMT (risk ratio $=0.83$, 95\% CI: $0.69-0.99 ; p=0.04$ ) with a NNT of $31 .^{25}$

\section{REPERFUSION TREATMENTS IN THE EXTEND- ED THERAPEUTIC TIME WINDOWS}

A European survey of national scientific societies and stroke experts from 44 countries reported mean rates of IVT of $7.5 \%$ and MT of $1.9 \%$ during the years $2015-2016$, with the highest rates being $20.6 \%$ and $5.6 \%$, respectively. ${ }^{26}$ The main reasons for not offering reperfusion therapies to most AIS patients include presentation beyond the eligible time windows and unknown time of symptom onset, comprising together the $66 \%$ of the non-treated cases. ${ }^{4}$ However, infarct growth rate is highly variable, with almost half of cases with LVO displaying 'favorable cerebral perfusion profiles' that may preserve tissue viability for several hours after the initial arterial occlusion. ${ }^{27}$ Therefore, recent RCTs utilized advanced brain imaging to capture and treat cases with late time window presentation and favorable cerebral perfusion profiles. Significant benefit from IVT has been demonstrated for selected cases with unknown time of onset or onset up to 9 hours. ${ }^{7,8}$ Similarly, patients with LVO presenting up to 16 to 24 hours after stroke onset and fulfilling specific imaging or clinical/ imaging mismatch criteria consistent with the detection of significant penumbra volumes and relatively small ischemic core volumes, may gain large outcome benefits from MT. ${ }^{9,10}$

It is evident that imaging, and particularly advanced imaging, is constantly becoming more crucial for the management of AIS. The depiction of diffusion weighted imaging/fluid-attenuated inversion recovery mismatch in brain magnetic resonance imaging (MRI) places stroke onset within the first 4.5 hours, whereas computed tomography (CT) or MRI perfusion imaging identifies the ischemic core and the critically, but not irreversibly, hypoperfused brain areas. ${ }^{28}$ Therefore, perfusion imaging may improve diagnostic accuracy, enhance outcome prediction, and enable patient selection for reperfusion therapies particularly in the late time windows. In this way, AIS management is moving towards a more individualized medical approach. ${ }^{29} \mathrm{On}$ the other hand, by increasing rates of reperfusion therapies and including cases with late presentation, AIS management becomes increasingly more demanding and complicated, given the fact that time is always an opponent in the race of a rapid, successful, and safe arterial reperfusion. ${ }^{30}$ Close monitoring of patients during AIS management is crucial and TCD may serve as an extension to the neurological assessment, guiding treatment decisions and refining CT-angiography/ MR-angiography (CTA/MRA) findings.

\section{TCD/TCCD APPLICATIONS IN ACUTE ISCHEMIC STROKE DIAGNOSIS}

TCD incorporates several key advantages in the assessment of AIS patients, since it is a low cost, bedside, non-invasive, easily repeatable, and safe (no-irradiation exposure) examination. Its diagnostic yield includes information about the site and degree of vascular occlusion, the collateral status and the detection of vascular steal phenomena, impaired cerebral hemodynamics, and real time embolization (Table 2). $\cdot^{1,32}$

Despite its inherent strengths, TCD examination and monitoring has some shortcomings (Table 2). Firstly, reliability of the examination depends greatly on the expertise of the operator. More importantly, $10 \%$ to $15 \%$ of individuals lack adequate transtemporal acoustic windows. In such cases, the use of contrast agents is indicated to improve monitoring and assist 
in decision making, with the cost of excluding from the diagnostic arsenal the detection of HITS. Another limitation is that only downstream monitoring of flow in vertebrobasilar occlusions treated with MT is feasible. Moreover, to avoid treatment delays, the presence of an additional individual from the stroke team, in charge of performing ultrasounds, is mandated. Obviously, continuous TCD monitoring, via a head frame or manually, may agitate severely affected stroke patients, something that is particularly important during endovascular procedures. Lastly, the nonradiolucent parts of the head frame can interfere with the lateral projection of the angiography and therefore limit its diagnostic accuracy. ${ }^{31,32}$

When performed urgently at the patient's bedside, TCD, in conjunction with carotid/vertebral artery duplex, may aid in the selection of candidates for IVT (Table 3). Although NIHSS scores $>10$ are consistent with LVO, cases with low NIHSS scores, even lower of 4, are not uncommon. ${ }^{33,16}$ TCD may rapidly detect cases with LVO causing a minor stroke, a finding that might encourage tissue plasminogen activator (tPA) administration without further delays to reduce the likelihood of late clinical deterioration. ${ }^{34}$ In such cases, LVO detection may also prompt some stroke experts to select tenecteplase as thrombolytic instead of alteplase, based on preliminary data showing higher recanalization rates with tenecteplase in comparison to alteplase. ${ }^{35}$

Patients with acute basilar artery occlusion may present with coma. A rapid and accurate ultrasound diagnosis will lead to earlier IVT infusion initiation that might improve odds of early recanalization, and subsequently outcomes. ${ }^{36}$ Accordingly, TCD has shown high accuracy (93\%) compared to angiography for emergent assessment of posterior circulation stroke. ${ }^{37}$ In addition, TCD may contribute to the differential diagnosis of stroke mimics thus limiting unnecessary treatments..$^{38}$

TCD is also helpful for the diagnosis of uncommon causes of AIS with contraindications to IVT. Representative examples include aortic dissection, intracranial

TABLE 2. TCD advantages and limitations in acute ischemic stroke diagnosis and monitoring

\begin{tabular}{|c|c|}
\hline Advantages & Limitations \\
\hline Bedside examination & Absent transtemporal windows (10-15\%) \\
\hline Easily repeatable & Useful only in the hands of specially trained and experienced operators \\
\hline Non-invasive & $\begin{array}{l}\text { Additional individual required to perform TCD during acute AIS man- } \\
\text { agement to avoid treatment delays }\end{array}$ \\
\hline Inexpensive & Can only monitor downstream flow in VB occlusions treated with MT \\
\hline Absence of irradiation & May agitate severely affected stroke patients during IA procedures \\
\hline $\begin{array}{l}\text { Provides diagnostic information about location and degree } \\
\text { of occlusion, collateral status, impaired hemodynamics, } \\
\text { vascular steal phenomenon, real-time embolization }\end{array}$ & $\begin{array}{l}\text { The nonradiolucent parts of the head frame can interfere with the lateral } \\
\text { projection of the angiography }\end{array}$ \\
\hline Refines CTA/MRA findings & \\
\hline
\end{tabular}

TABLE 3. TCS selection of appropriate candidates for IVT

Assist in treatment decisions for patients presenting with mild stroke $(\mathrm{NIHSS}<4)$

Assist in treatment decisions in comatose patients (BA occlusion)

Assist in differential diagnosis of stroke mimics

Assist in timely diagnosis of uncommon causes of AIS with contrandications to IVT (aortic arch dissection, endocarditis, intracranial aneurysm with intraluminal thrombus, vasospasm due to undiagnosed $\mathrm{SAH}$ )

TCS; transcranial sonography, IVT; intravenous thrombolysis, NIHSS; National Institute Health Stroke Scale, BA; basilar artery, AIS; acute ischemic stroke, $\mathrm{SAH}$; subarachnoid haemorrhage. 
aneurysm with intraluminal thrombus, vasospasm due to undiagnosed subarachnoid hemorrhage, and endocarditis which may be suspected by the detection of a high microembolic signal load..$^{39-42}$ Fig. 1 presents a case with left middle cerebral artery (MCA) stroke due to aortic dissection extending into the left common carotid artery and the left subclavian artery. Rapid ultrasound diagnosis of aortic dissection averted treatment with IVT.

The information provided by neurosonology regarding location of LVO may be complimentary to other imaging modalities including CTA and MRA. It should be noted that specific contraindications exist for the other established neuroimaging methods that are currently used for assessment of recanalization status (eg, pacemakers for MRA, renal insufficiency for CTA). Notably, in a study comparing neurosonology with correlative angiography (MRA or CTA) in 58 AIS patients, MRI was inconclusive or not possible because of extensive movement artifacts or contraindications in $19 \%$ of the study patients. ${ }^{43}$ In additional $14 \%$ critically ill patients, MRI could not be performed because of insufficient ability to monitor vital parameters in the scanner. In contrast, ultrasound examination was possible and conclusive in $93 \%$ of the studied cases. ${ }^{43}$

Finally, transcranial ultrasound emerges as a useful tool in the prehospital diagnosis of AIS in ambulances (mobile stroke units) or helicopters. More specifically, there are preliminary studies arguing in favor of the feasibility and high diagnostic accuracy of emergency transcranial ultrasound assessment combined with neurological examinations for major AIS detection. ${ }^{44,45}$ In the context of pre-hospital application, TCD optionally combined with ultrasound perfusion imaging has the potential to identify LVO patients which should be transferred directly to an interventional stroke center. ${ }^{46}$

\section{TCD/TCCD APPLICATIONS IN MONITORING OF RECANALIZATION DURING ACUTE REPER- FUSION THERAPIES}

Emergent TCD/TCCD examination of patients with AIS serves for both LVO detection and monitoring of recanalization. Use of a bedside, fast track protocol with full power, high pulse repetition frequencies (PRF) and gate $>10 \mathrm{~mm}$ is recommended for the detection of LVO ${ }^{47}$ Residual flow at the site of the occlusion has to be identified and quantified by using the thrombolysis in brain ischemia (TIBI) grading system (Fig. 2).$^{48}$ Thereafter, the transducer can be fixed over the temporal bone window with a standard head frame (Table 4). ${ }^{49}$
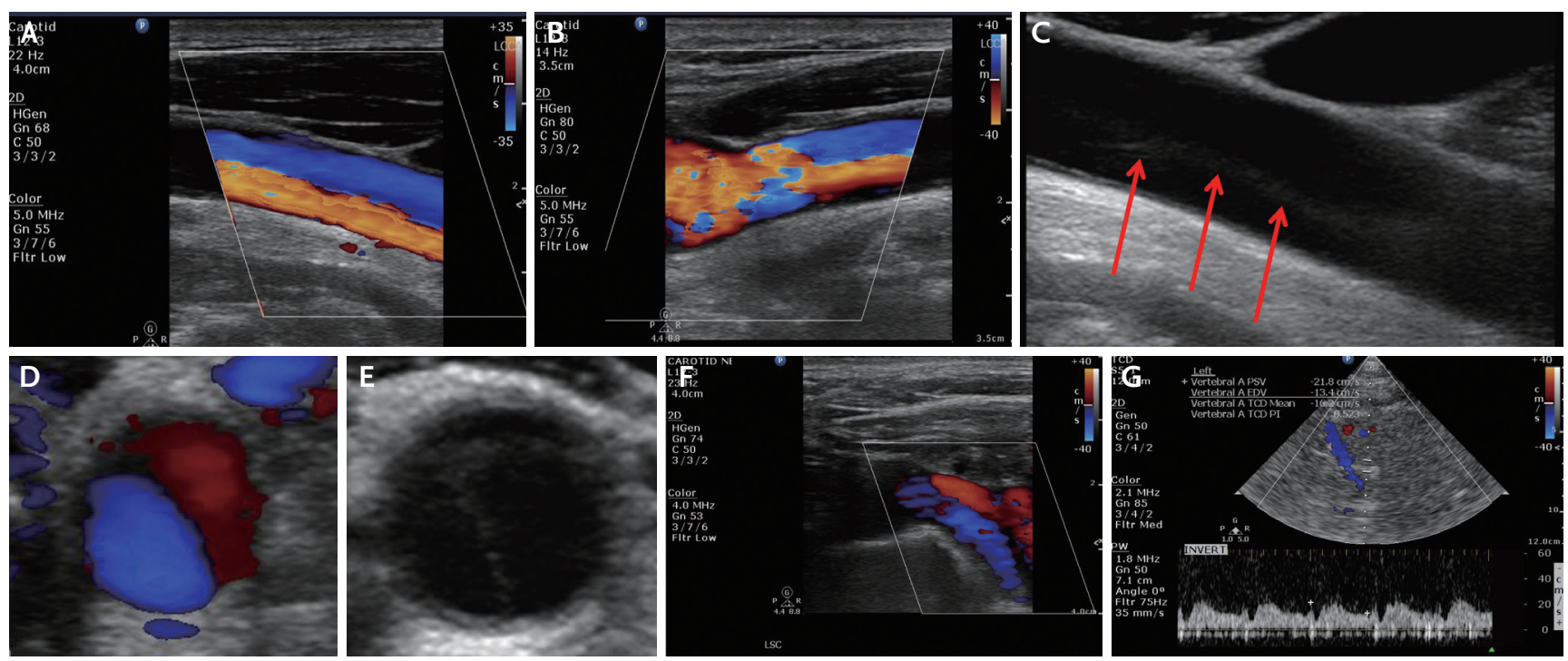

FIG. 1. A 53-year-old patient presenting with dysarthria and right arm weakness due to acute ischemic stroke caused by aortic dissection extending into the left common carotid and subclavian arteries. (A-C) Longitudinal and (D, E) transverse duplex and B-mode images showing double lumen and intimal flap in left common carotid artery. (F) Double lumen appearance was also documented in the left subclavian artery. (G) Transcranial color-coded duplex revealing low flow velocities and systolic deceleration in the left middle cerebral artery. 
Most importantly, the investigators of safe implementation of thrombolysis in Stroke-International Stroke Thrombolysis Register registry have documented that TCD monitoring of recanalization in AIS patients treated with IVT is not associated with time delays and also may be related to improved clinical outcomes..$^{\circ}$

Numerous studies have underscored the importance of early recanalization in AIS patients with underlying LVO..$^{1-53}$ Several TCD findings have been proposed as predictors of successful recanalization after IVT (Table 5, Fig. 3). Residual flow, as expressed with the TIBI grading system, at the site of the LVO before tPA infusion, has been correlated with the likelihood of complete recanalization. Indeed, an international multi-center study reported a wide range of recanalization rates, from $18 \%$ in patients with TIBI grade o to $48 \%$ in those with TIBI grade 3. Absence of flow, with TIBI grade $\mathrm{O}$, was associated with low rates of recanalization, but also with delayed recanalization and poor outcomes. ${ }^{54} \mathrm{In}$ another study, patients with LVO receiving systemic or intra-arterial tPA infusion underwent continuous TCD monitoring. End diastolic velocity but not peak systolic velocity increase correlated with early neurological improvement, but also with favourable functional outcome at 3 months..$^{55}$ Moreover, in a small patient series with MCA related AIS, microembolic signals (MES) detected on TCD distal to the high-grade stenosis or near-occlusion predicted complete, spontaneous or post-throm-

TABLE 4. TCD settings for monitoring of recanalization

\begin{tabular}{l} 
Portable TCD and duplex \\
Fast track protocol \\
Use full power \\
High PRF, gate $\geq 10 \mathrm{~mm}$ \\
Occlusion location/worst residual flow: use TIBI grading \\
Monitoring set \\
Hand-held monitoring in agitated patients \\
\hline
\end{tabular}

TCD; transcranial Doppler, PRF; pulse repetition frequency, TIBI; thrombolysis in brain ischemia.
TIBI 0

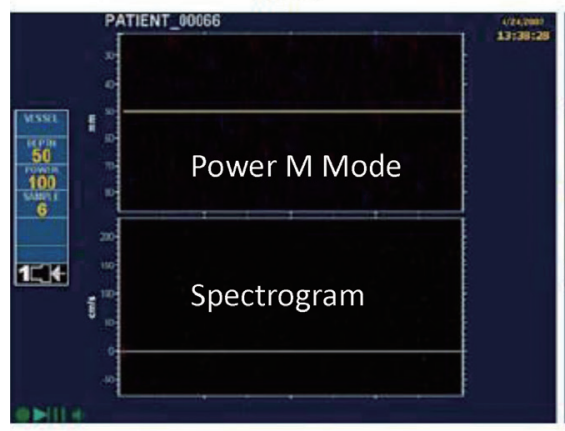

TIBI 3

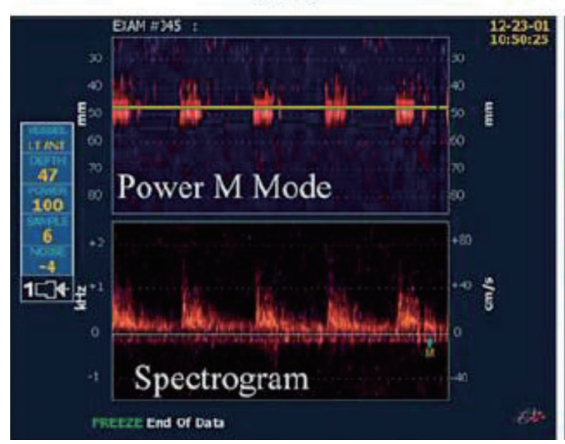

TIBI 1

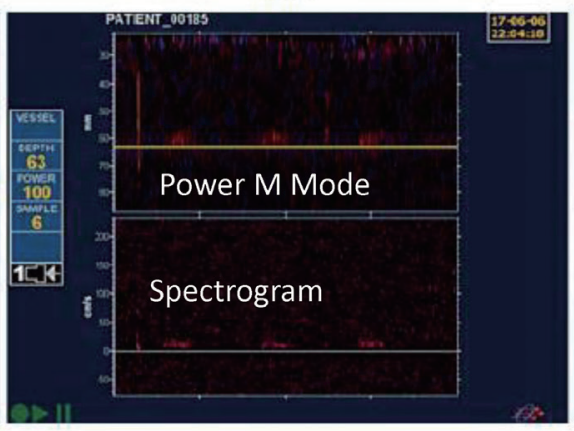

TIBI 4



$\mathrm{TIBI} 2$

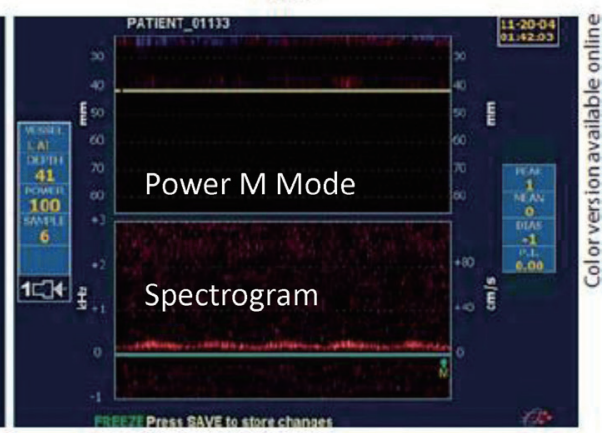

TIBI 5

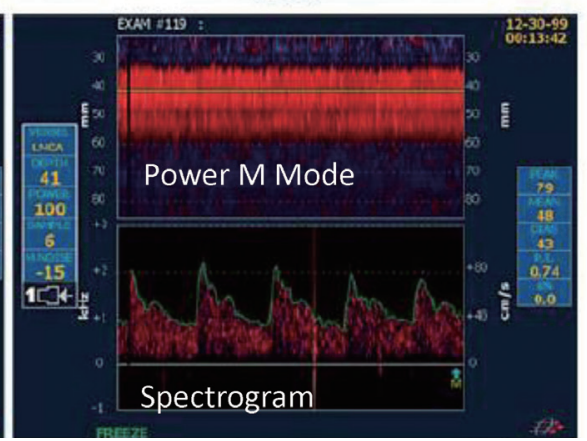

FIG. 2. Thrombolysis in brain ischemia (TIBI) grading for the assessment of the degree of arterial occlusion. TIBI o: complete absence of residual flow, TIBI 1: minimal flow with only systolic spikes, TIBI 2: blunted waveform with flattened systolic upstroke, TIBI 3: dampened flow with decreased mean flow velocities by $>30 \%$ compared to contralateral side, TIBI 4: stenotic waveform with acceleration of mean flow velocities $(>80 \mathrm{~cm} / \mathrm{s}$, or $>30 \%$ increase compared to contralateral vessel), $\mathrm{TIBI} 5$ : normal flow. The upper and lower panels correspond to power $\mathrm{M}$ mode and spectral display (spectrogram) displays respectively. 
bolysis, recanalization, indicating that MES may signal ongoing clot dissolution (Fig. 4). ${ }^{56}$ Good collateral sta-

TABLE 5. TCD predictors of recanalization during monitoring of IVT for AIS

Residual TIBI grade flow at the site of the LVO

Increase in EDV

Presence of microembolic signals distal to the location of occlusion

Presence of flow distal to the location of occlusion

Absence of tandem occlusion

Good collateral status (flow diversion in ACA/PCA, retroperfusion of distal MCA via leptomeningeal collaterals)

TCD; transcranial Doppler, IVT; intravenous thrombolysis, AIS; acute ischemic stroke, TIBI; thrombolysis in brain ischemia, LVO; large vessel occlusion, EDV; end diastolic velocity, ACA; anterior cerebral artery, PCA; posterior cerebral artery, MCA; middle cerebral artery. tus, reflected by flow diversion in the anterior cerebral artery/posterior cerebral artery and/or retroperfusion of the distal MCA via leptomeningeal collaterals, is also a positive predictor of recanalization..$^{57}$ Indeed, in an endovascular therapy study, pre-treatment good collateral status was associated with higher recanalization rates following intra-arterial thrombolytic therapy or MT, with or without preceding IVT..$^{8}$ Conversely, negative prognostic factors of $\mathrm{tPA}$-induced recanalization include complete absence of residual flow distal to the site of occlusion and presence of tandem or terminal internal carotid artery occlusion..$^{9}$

TCD monitoring may also assess speed of recanalization in cases with AIS due to LVO. Interestingly, speed of clot lysis is associated with more pronounced early clinical improvement. Fast/sudden recanalization leads more frequently to complete clot lysis than stepwise or slow recanalization. Slow or partial recanalization (TIBI

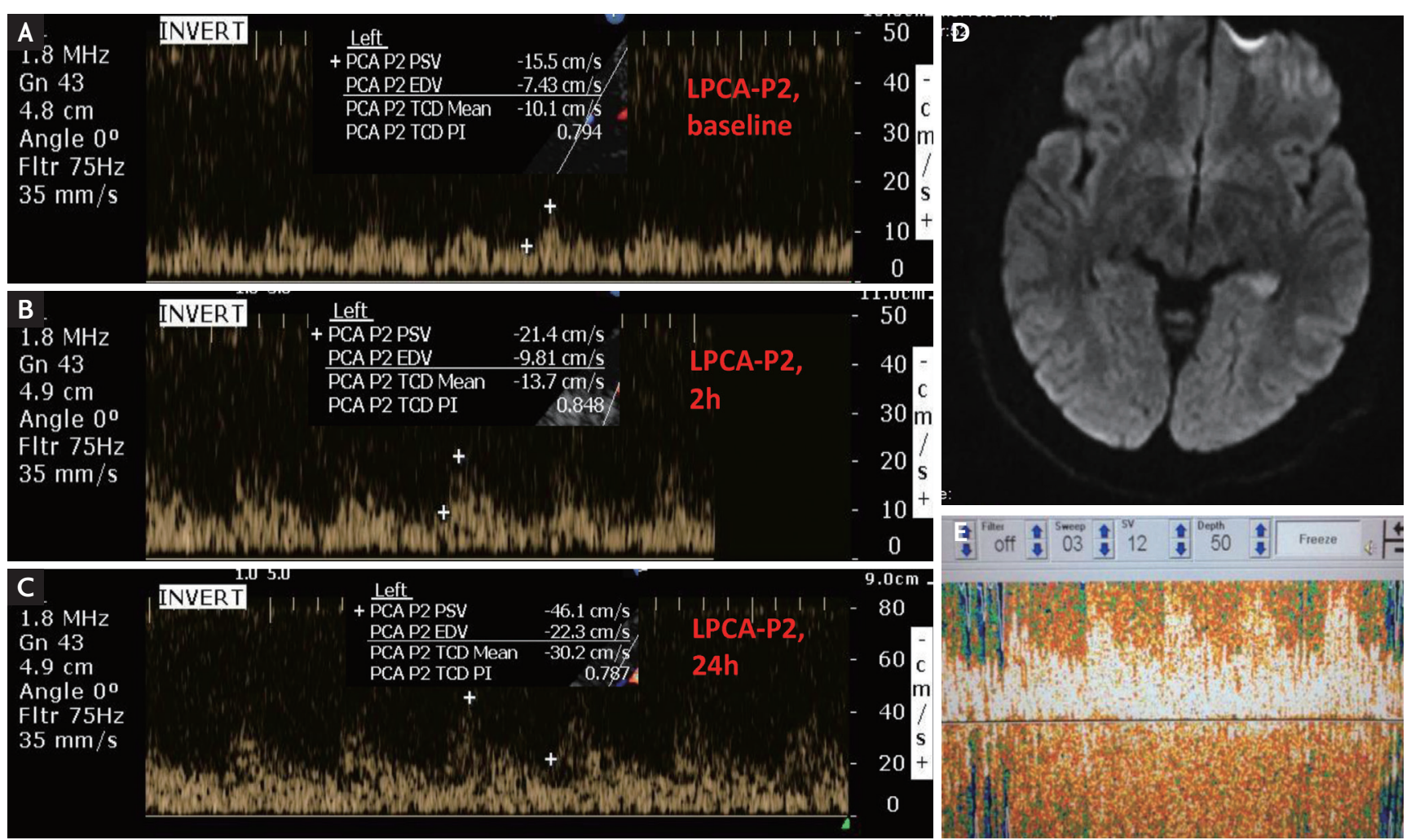

FIG. 3. A 38-year-old woman presented with acute onset of right homonymous hemianopia. (A) Baseline Transcranial Doppler (TCD) depicted occlusion of the $\mathrm{P}_{2}$ segment of the left posterior cerebral artery (PCA), with residual flow thrombolysis in brain ischemia (TIBI) grade II. (B) Two hours after tissue plasminogen activator infusion a mild improvement in the waveform of the left PCA (TIBI grade III) was noted, (C) which was more evident 24 hours later showing complete recanalization TIBI grade V, with complete resolution of the neurological deficits. (D) Follow up brain diffusion weighted magnetic resonance imaging showing a small left occipital acute ischemic lesion. (E) TCD bubble study revealed a high grade right-to-left shunt after Valsava maneuver indicative of large functional patent foramen ovale. 
3) are negative outcome predictors. ${ }^{47}$ Apart from speed, timing of recanalization is also associated with better short-term and long-term outcomes. A recent TCD monitoring study showed that every 15 -minute delay in recanalization reduces odds of favourable functional outcome by $16 \%$ (95\% CI: 3-37\%). Accordingly, symptom onset to treatment initiation is linked to successful reperfusion, since every 10-minute delay in IVT infusion was associated with delays of 1.3 minutes between tPA-bolus and onset of recanalization..$^{36}$

The validity of TCD for monitoring intra-arterial reperfusion therapies has been assessed in an international, multicentre study. Consecutive AIS patients with LVO underwent intra-arterial reperfusion procedures with simultaneous real-time TCD-monitoring. Residual flow signals at the site of angiographically confirmed occlusions were monitored at a constant transtemporal insonation angle using a standard head-frame. Recanalization was assessed simultaneously by digital subtraction angiography (DSA) and ultrasound using thrombolysis in myocardial infarction (TIMI) and TIBI criteria, respectively. Independent readers blinded to DSA findings performed validation of TIBI flow grades. The interrater reliability for estimating TIBI grades and recanalization was satisfactory (Cohen $\kappa: 0.838$ and 0.874 , respectively; $p<0.001)$. The accuracy parameters for detection of complete recanalization (TIBI grades IV or V) using TCD were high compared to the gold standard of DSA (TIMI grade III); sensitivity: 88\% (95\% CI: 72-96\%), specificity: 89\% (95\% CI: 79-95\%), positive predictive value: $81 \%$ (95\% CI: 65-91\%), negative predictive value: 93\% (95\% CI: 84-98\%), and overall accuracy 89\% (95\% CI: $80-94 \%) .{ }^{60}$ Similarly, another TCCD study examining LVO patients receiving IVT who subsequently underwent intra-arterial thrombolysis if no recanalized was detected during TCD monitoring of tPA infusion, showed also high concordance rates between TIBI and TIMI scores. ${ }^{61}$

Continuous TCD or TCCD monitoring during endovascular reperfusion procedures detects different types
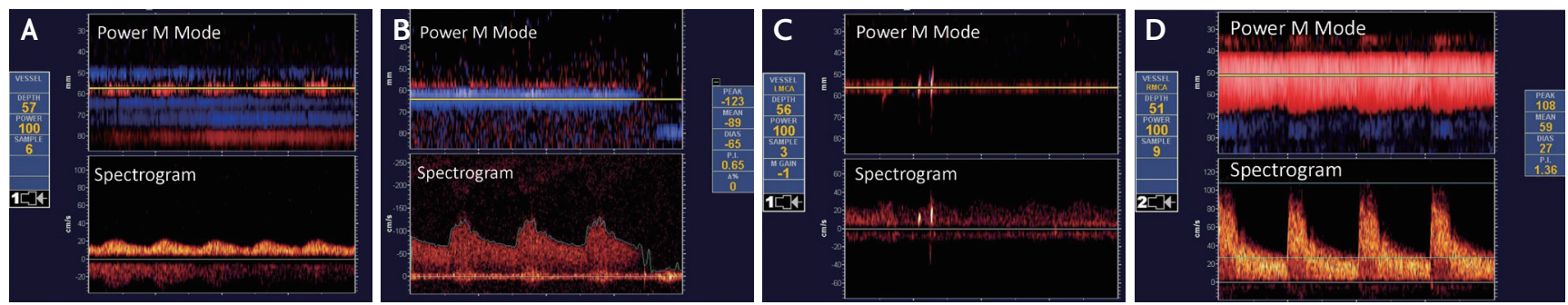

FIG. 4. A 74-year-old woman with acute onset of dysarthria and right arm weakness and NIHSS-score of 10. (A) Initial TCD showed occlusion with TIBI grade II of the left middle cerebral artery (MCA) and (B) normal flow spectrum of the right MCA. The patient received tPA 4 hours and 19 minutes after symptom onset. (C) Twenty-five minutes after bolus injection TCD monitoring depicts microembolic signals within the blunted flow spectrum of the left MCA. (D) Two hours later a complete recanalization (TIBI grade $\mathrm{V}$ ) is observed in the left MCA. The patient shows significant clinical improvement with NIHSS-score of 5 at two hours following tPA-bolus. The upper and lower panels correspond to Power M Mode and spectral display (spectrogram) displays respectively.

TABLE 6. Clinically relevant TCD-monitoring signatures during intra-arterial procedures

\begin{tabular}{ll}
\hline Events & \multicolumn{1}{c}{ Sonographic findings } \\
\hline Recanalization & From TIMI o/I to II: MFV increase by $19 \pm 16 \mathrm{~cm} / \mathrm{s}$ \\
& From TIMI o/I to III: MFV increase by $65 \pm 39 \mathrm{~cm} / \mathrm{s}$ \\
Arterial re-occlusion & Worsening of TIBI grade on TCD by $\geq 1$ \\
Air embolization & Frequent HITS with higher intensity \\
Artery-to-artery embolization & HITS at the end of the intra-arterial procedure \\
Hyperperfusion & MFV increase of $\geq 1.5$ times compared to the contralateral artery \\
\hline
\end{tabular}

TCD; transcranial Doppler, TIMI; thrombolysis in myocardial infarction, MFV; mean flow velocities, TIBI; thrombolysis in brain ischemia; HITS; high intensity transient signals. 
of flow changes of potential diagnostic value (Table 6). Rubiera et al. ${ }^{62}$ applied continuous TCD monitoring in 51 AIS patients undergoing intra-arterial reperfusion procedures that included intra-arterial thrombolysis, mechanical thrombectomy using Merci retriever device or Penumbra aspiration system deployment, balloon angioplasty, and stenting. During these procedures, the following flow findings emerged: contrast injection produced high-intensity transient signals (HITS) and median artificial mean flow velocities (MFV) increase of 10.5\%; similarly, Merci retriever advancement, thrombus engagement, and traction resulted in HITS of alternating direction with transient, artificial median MFV decrease of 11.5\%; Penumbra passes produced HITS of lower intensity than those of Merci system as well as greater transient decrease of MVF; intra-arterial thrombolysis produced MFV increase of $7.5 \%$ and HITS from the infusion of saline; catheter deployment and manipulations gave rise to HITS with characteristic harmonic "halo-like" bruits within the flow spectra, in both flow directions.

Recanalization produced increases in flow velocities as follows: MFV increased by $19 \pm 16 \mathrm{~cm} / \mathrm{s}$ for recanalization increasing TIMI $^{63}$ grades o or I to TIMI II, and by $65 \pm 39 \mathrm{~cm} / \mathrm{s}$ for recanalization increasing TIMI grades o or I to TIMI III. Similarly, end-diastolic velocities increased by $14 \pm 13 \mathrm{~cm} / \mathrm{s}$ and $45 \pm 21 \mathrm{~cm} / \mathrm{s}$, respectively. Worsening of TIBI grade on TCD by $\geq 1$ was regarded as diagnostic of arterial re-occlusion. Although infrequent, air emboli might appear during catheter manipulations, and in these cases TCD captured frequent HITS with higher intensity than those of solid emboli. This potentially harmful event prompts repositioning of the sheath connector. Real time artery-to-artery embolization was detected as HITS at the end of the intra-arterial procedure and was typically accompanied by reformation of intraluminal thrombi. Finally, successful recanalization might result in cerebral hyperperfusion with MFV increase of $\geq 50 \%$ compared to the contralateral artery. Such findings may prompt intensive blood pressure reductions. ${ }^{62}$

Interestingly, a recent Italian study has reported similar findings. Serial TCCD examinations assessed the vessel status and cerebral hemodynamics of consecutive patients with acute anterior circulation LVO soon after, at 48 hours after, and 1 week after endovascular reperfusion procedures. Successful recanalization and normal peak systolic velocity (PSV) ratio (PSV of recanalized middle cerebral artery/PSV of contralateral middle cerebral artery $<1.2$ ) at 48 hours and after 1 week from endovascular therapies emerged as independent predictors of good outcome at 3 months. Patients who subsequently developed postinterventional intracranial hemorrhage (ICH) showed a higher mean PSV ratio (3.5 \pm 0.2 vs. $2.4 \pm 0.1, p<0.0001)$ soon after successful recanalization. In multivariate analysis, increasing early PSV ratio was independently associated with an eight-fold higher odds of post-procedural ICH. The investigators concluded that peri- and post-procedural ultrasound monitoring of LVO patients might be an effective bedside method for assessing treatment efficacy, shedding light on outcome variability and identifying patients at increased risk of ICH complicating endovascular reperfusion procedures. ${ }^{64}$

Therefore, the implementation of TCD monitoring during intra-arterial procedures might provide with some clinically relevant information that may modify treatment decisions. In the hands of an experienced sonographer, the previously descripted sonographic signatures can be reliably differentiated into clinically irrelevant or important. Moreover, certain potentially harmful events, such as air embolization, artery-to-artery embolization, and hyperperfusion may only be captured by serial ultrasound assessments.

\section{TCD/TCCD APPLICATIONS IN DETECTING OF CAUSES OF EARLY NEUROLOGICAL DETERIO- RATION FOLLOWING REPERFUSION THERA- PIES}

Following IVT up to $14 \%$ of patients might experience early neurological deterioration. ${ }^{64}$ sICH, malignant cerebral edema, persistent occlusion after IVT in AIS with underlying LVO, expansion of ischemic infarct, arterial re-occlusion, collapse of the collateral flow, clot extension, and early recurrent ischemic stroke in previously unaffected territory are the most common causes of early neurological deterioration. ${ }^{65-67}$ A single center study reported rates of early neurological deterioration, within 72 hours, of $30 \%$. The mechanisms included progression of the ischemic infarct, brain edema and 
sICH. Expansion of the ischemic infarct was associated with large artery atherosclerosis, indicating that failure to recanalize or early re-occlusion may represent the main causes of infarct progression. ${ }^{68}$

TCD monitoring may identify several mechanisms of early neurological deterioration after acute reperfusion therapies (Table 7). Monitoring of acute ICH growth with transcranial duplex sonography has been shown to be both feasible and reliable. A TCCD/CT correlation study in 34 patients with ICH found excellent results regarding the concordance between transcranial sonography and CT measurements of hematoma diameters in longitudinal, sagittal and coronal planes. Apparently, TCCD was efficient in detecting early hematoma

TABLE 7. TCD identification of causes of early neurological deterioration in AIS treated with IVT

\section{$\mathrm{sICH}$}

Malignant cerebral edema-increased ICP (MDS)

Failure to recanalize in LVO

Arterial reocclusion

Collateral failure

TCD; transcranial Doppler, AIS; acute ischemic stroke, IVT; intravenous thrombolysis, $\mathrm{SICH}$; symptomatic intracranial haemorrhage, ICP; intracranial pressure, MDS; midline shift, LVO; large vessel occlusion. growth. The correlation was also good for estimating total hematoma volume. The study included patients with both deep or lobar ICH. ${ }^{69}$ Subsequently, smallscale studies have shown high sensitivity $(\geq 90 \%)$ and specificity $(\geq 95 \%)$ of TCCD for the depiction of hemorrhagic transformation of AIS. ${ }^{70,71}$ Fig. 5 shows a case of sICH complicating IVT for AIS that was diagnosed at the beside using TCCD when a 12-point increase in NIHSS-score was noted during serial neurological evaluations.

Arterial re-occlusion is a common pathophysiological mechanism responsible for early neurological worsening after reperfusion therapies for AIS. It occurs in $15-34 \%$ of IVT treated patients and it accounts for the $2 / 3$ of cases experiencing deterioration after initial improvement. ${ }^{72}$ Stroke severity and large artery atherosclerotic stroke have been identified as independent predictors of re-occlusion following IVT. ${ }^{73}$ Early re-occlusion after MT might be observed in $2.3-7.2 \%$ of cases. Age, pre-admission statins, intracranial internal carotid artery occlusion, IVT treatment, number of passes during MT, atherosclerotic cause of occlusion, high numbers of platelets or high levels of D-dimers on admission, residual embolic fragments or stenosis at the thrombectomy site, and stent implantation are factors associated with early re-occlusion. ${ }^{74-76} \mathrm{TCD}$ monitoring can reliably detect in real time arterial
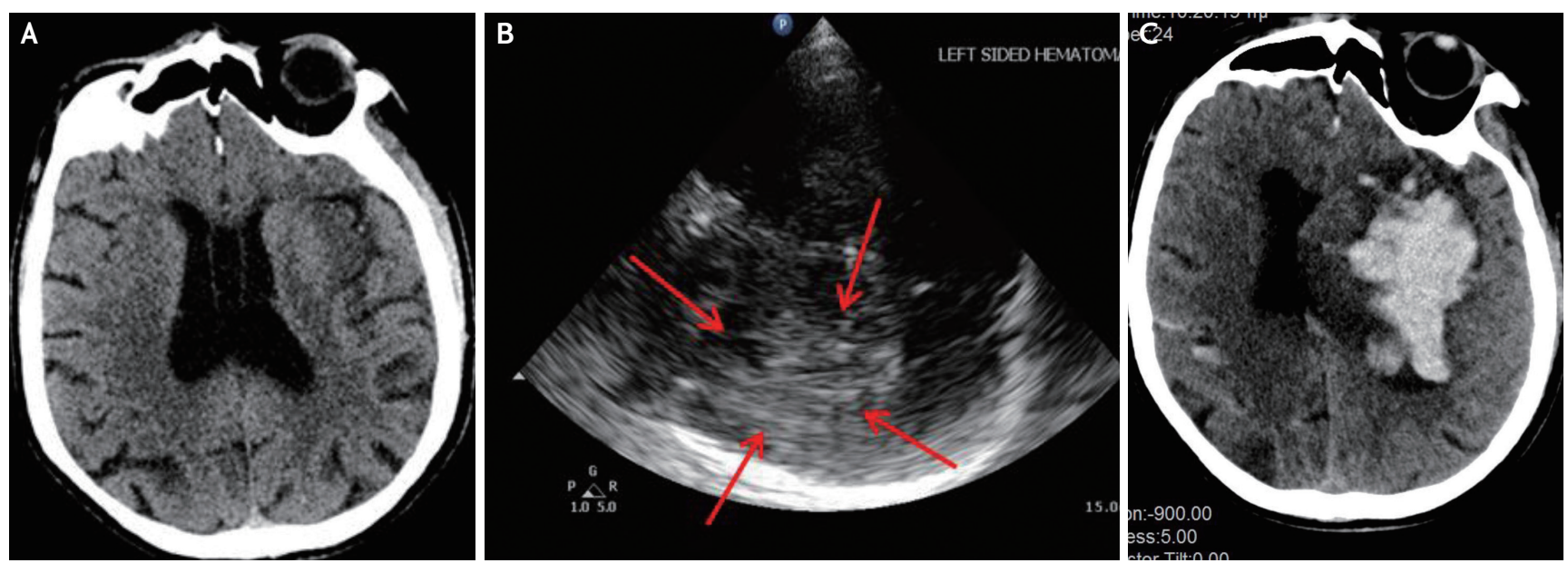

FIG. 5. A 80-year-old man presented with acute onset of expressive aphasia and right hemiparesis (National Institutes of Health Stroke Scale [NIHSS]-score of 7). (A) Admission brain computed tomography (CT) disclosed an Alberta Stroke Program Early CT Score of 9/10 with hypodense insular ribbon. The patient received tPA at 4 hours following symptom onset, and 4 hours after treatment a clinical deterioration was noticed ( $\mathrm{NI}$ HSS-score 19). (B) Transcranial color-coded duplex (TCCD) sonography depicted a hyperdense mass (arrows) within the left hemisphere (C) that corresponded to a large hematoma within the ischemic area, as shown on the follow-up brain CT. TCCD evaluation at the bedside assisted in the early recognition of symptomatic intracranial haemorrhage as the underlying cause of early neurological deterioration in this patient. 
re-occlusion, and therefore provide explanation for early neurological deterioration. ${ }^{7377}$

Cerebral blood steal phenomenon has been described as a cause of early neurological deterioration in patients with large vessel occlusions. The phenomenon of paradoxical reduction in the perfusion of the affected hemisphere under cerebral vasodilatory stimuli due to intracranial steal from the non-affected hemisphere is called Reverse Robin Hood syndrome. ${ }^{78}$ It can be easily reproduced and recorded with the breath holding test, where a decrease in blood flow velocities in the affected side is detected under hypercapnia. Male gender, younger age, persisting arterial occlusions, and excessive sleepiness have been associated with the Reverse Robin Hood syndrome. ${ }^{79}$

\section{TCD/TCCD APPLICATIONS IN THE PROGNOSIS OF CLINICAL OUTCOMES OF AIS PATIENTS TREATED WITH ACUTE REPERFUSION THERA- PIES}

The introduction of TCD or TCCD monitoring examination in AIS management provides valuable information concerning response to treatment and outcome prognosis. Residual flow, as assessed by TIBI grades, predicts likelihood of recanalization after IVT but also outcomes, in a way that patients showing absence of recanalization with TIBI grades $\mathrm{O}-2$ experience poor prognosis in more than $50 \%$ of cases. Additional independent predictors of poor outcome are levels of admission systolic blood pressure and glucose, and stroke severity ${ }^{54}$ Another study reported sensitivity of $57.7 \%$ and specificity of $87.2 \%$ for complete recanalization to predict good outcome at 3 months. ${ }^{80}$ TCD assessment of recanalization appears as the strongest predictor of favorable functional outcome (mRS-scores of o-1) at 3 months. ${ }^{81}$ Furthermore, a small single center study of serial TCD examinations showed that delayed reperfusion ( $>6$ hours) or persisting occlusion after IVT infusion has a higher risk of parenchymal hematoma development. ${ }^{82}$ The results were also replicated by a larger multi-center study that found a six-fold increase of the risk of sICH after IVT in cases without recanalization. ${ }^{53}$

Selection of patients with LVO for rescue intra-arterial reperfusion procedures is assisted with TCD exam- ination and monitoring. An interesting study looked for potential sonographic findings that may predict inadequate response to IVT, and therefore the need for rescue intra-arterial intervention. An affected MCA to contralateral MCA mean flow velocity ratio $<0.6$ had a sensitivity of $94 \%$ (95\% CI: 63-99\%), specificity of 100\% (97.5\%; lower CI of 54\%), positive predictive value of 100\% (lower CI: 80\%); and negative predictive value of 86\% (CI: $42-99 \%$ ) for identifying LVO in the anterior circulation requiring intra-arterial reperfusion procedure. Additionally, none of the patients with complete absence of flow in the MCA showed signs of clot lysis after IVT. ${ }^{83}$ Another small scale study showed that recanalization with TIBI grades of 3 to 5 within 30 minutes of IVT initiation resulted in favorable outcomes in 59\% of cases, and thus may serve as a 'reperfusion marker' before transfer of the patient to the angio-suite. ${ }^{61}$ Therefore, and in accordance with the latest guidelines on MT in AIS patients due to LVO that highlight the importance of not waiting for the effect of IVT but rather administer alteplase bolus and transfer the patients directly to the angiography suite to swiftly initiative endovascular procedures, TCD may detect early those cases with early tPA-induced recanalization averting MT. $^{12}$

After successful recanalization of LVO, MES detection may predict stroke recurrence. Interestingly, a TCD study of 111 patients that underwent MT with complete recanalization found that MES detection was correlated with new embolic events with almost seven-fold increased risk of recurrent stroke. ${ }^{84}$ Therefore, detection of MES early post-reperfusion may prompt the administration of more potent antithrombotic therapies to reduce risk of stroke recurrence. ${ }^{85}$

Absence of recanalization after IVT treatment for LVO-associated AIS increases risk of not only sICH but also of malignant edema development with a subsequent increase of intracranial pressure (ICP). The Systemic thrombolysis in patients with acute ischemic stroke and Internal Carotid ARtery Occlusion study demonstrated that, although IVT increased odds of favorable outcome (adjusted odds ratio: 1.80; 95\% CI: 1.03-3.15; $p=0.037$ ), it also increased rates of malignant edema (8.3\% vs. $3.1 \%) .{ }^{86}$ Several studies, although using different methodological models, have investigated the accuracy of non-invasive, TCD-based estimation 
of ICP levels. ${ }^{87}$ These methods are based on TCD-derived pulsatility index $(\mathrm{PI})$ values, non-invasive cerebral perfusion pressure (CPP) estimations, or mathematical models. The last two approaches use mainly measurements of arterial blood pressure and MFV to predict CPP and ICP values. The studies report accuracies for detection of ICP $\geq 20 \mathrm{mmHg}$ with an area under the curve ranging from 0.62 to 0.92 . The TCD-based methods may estimate ICP values with a range of approximately $\pm 12 \mathrm{mmHg}$. However, the most important utility of TCD-monitoring is perhaps the tracing of dynamic changes of ICP over time. ${ }^{87}$ The simplest way to indirectly monitor ICP levels is by estimating the PI and inspecting the flow spectra waveforms. Increase of ICP is accompanied by increase of PI, (provided that other factors influencing PI such as blood $\mathrm{CO}_{2}$ levels are relative stable), decrease in diastolic flow velocities, and sometimes even by flow reversal during the diastolic phase. ${ }^{49}$ In addition, TCCD is shown to depict midline shift with satisfactory accuracy. Motuel et al. ${ }^{88}$ estimated midline shift by measuring the difference between the distance from skull to the third ventricle on both sides and compared with measurements performed on brain CT. The area under the ROC curve for depicting midline shift with TCCD was 0.86 (95\% CI: 0.74-0.94), while by using $0.35 \mathrm{~cm}$ as a cut-off, the sensitivity, specificity, and positive likelihood ratio were $84.2 \%, 84.8 \%$ and 5.56, respectivelly.

Interestingly, a recent paper showed that after MT, TCD-monitoring guided blood pressure and intracranial pressure control was feasible and reduced early neurological deterioration of patients. Indeed, TCD identified cases with low flow velocities and intracranial hypertension, and subsequently prompt therapeutic interventions that improved outcomes. Early neurological deterioration and 3-month mortality rates were reduced in the group that underwent TCD monitoring and had abnormal findings, compared with the control group without TCD monitoring (13.8 vs. $37.5 \%, p=0.036$; O vs. $25.0 \%, p=0.012$ ). In multivariable analysis, TCD monitoring independently contributed to a reduced likelihood of early neurological deterioration after MT (adjusted OR: $0.267,95 \%$ CI: $0.074-0.955 ; p=0.042$ ). ${ }^{89}$

\section{CONCLUSIONS}

The main advantages of ultrasound in acute stroke management derive from its non-invasive nature, the repeatability and feasibility to perform at the bedside. Among all conventional imaging modalities, only TCD may detect solid or air particle real-time embolization, as well as diagnose hyperperfusion. Importantly, in cases with contraindications to CTA, as in severe renal insufficiency, TCD can reliably and rapidly screen for the site and degree of a possible LVO. Overall, TCD examination may serve as a useful/complementary tool for patient selection for IVT, while continuous TCD monitoring during tPA infusion or endovascular procedures can reliably detect recanalization in real-time. A possible arterial re-occlusion may also be easily captured in real time, whereas TCCD may rapidly depict sICH or cerebral edema development in cases with neurological deterioration. In the era of pre-hospital stroke management, TCD assessment within the ambulance or the helicopter has been shown to be feasible, fast, and with high sensitivity and specificity. Therefore, portable TCD examination could potentially assist in the rapid triage of stroke patients or serve as an additional diagnostic tool for future clinical trials involving new therapeutic agents.

\section{REFERENCES}

1. Alexandrov AV. Current and future recanalization strategies for acute ischemic stroke. J Intern Med. 2010;267:209-219.

2. Tsivgoulis G, Safouris A, Katsanos AH, Arthur AS, Alexandrov AV. Mechanical thrombectomy for emergent large vessel occlusion: a critical appraisal of recent randomized controlled clinical trials. Brain Behav. 2016;6:eoo418.

3. Asaithambi G, Tong X, Lakshminarayan K, Coleman King SM, George MG. Current trends in the acute treatment of ischemic stroke: analysis from the Paul Coverdell National Acute Stroke Program. J Neurointerv Surg. 2020;12:574-578.

4. Reiff T, Michel P. Reasons and evolution of non-thrombolysis in acute ischaemic stroke. Emerg Med J. 2017;34:219-226.

5. Tsivgoulis G, Katsanos AH, Schellinger PD, Köhrmann

M, Caso V, Palaiodimou L, et al. Advanced neuroimaging in stroke patient selection for mechanical thrombecto- 
my. Stroke. 2018;49:3067-3070.

6. Psychogios K, Magoufis G, Safouris A, Kargiotis O, Katsanos $\mathrm{AH}$, Spiliopoulos S, et al. Eligibility for intravenous thrombolysis in acute ischemic stroke patients presenting in the 4.5-9 h window. Neuroradiology. 2020;62:733-739.

7. Thomalla G, Simonsen CZ, Boutitie F, Andersen G, Berthezene Y, Cheng B, et al. MRI-guided thrombolysis for stroke with unknown time of onset. $N$ Engl J Med. 2018;379:611-622.

8. Ma H, Campbell BCV, Parsons MW, Churilov L, Levi $\mathrm{CR}$, Hsu C, et al. Thrombolysis guided by perfusion imaging up to 9 hours after onset of stroke. $\mathrm{N}$ Engl J Med. 2019;380:1795-1803.

9. Nogueira RG, Jadhav AP, Haussen DC, Bonafe A, Budzik RF, Bhuva P, et al. Thrombectomy 6 to 24 hours after stroke with a mismatch between deficit and infarct. N Engl J Med. 2018;378:11-21.

10. Albers GW, Marks MP, Kemp S, Christensen S, Tsai JP, Ortega-Gutierrez S, et al. Thrombectomy for stroke at 6 to 16 hours with selection by perfusion imaging. $N$ Engl J Med. 2018;378:708-718.

11. Ahmed N, Audebert H, Turc G, Cordonnier C, Christensen $\mathrm{H}$, Sacco S, et al. Consensus statements and recommendations from the ESO-Karolinska Stroke Update Conference, Stockholm 11-13 November 2018. Eur Stroke J. 2019;4:307-317.

12. Powers WJ, Rabinstein AA, Ackerson T, Adeoye OM, Bambakidis NC, Becker K, et al. Guidelines for the early management of patients with acute ischemic stroke: 2019 update to the 2018 guidelines for the early management of acute ischemic stroke: a guideline for healthcare professionals from the American Heart Association/American Stroke Association. Stroke. 2019;50:e344-e418.

13. Tsivgoulis G, Kargiotis O, Alexandrov AV. Intravenous thrombolysis for acute ischemic stroke: a bridge between two centuries. Expert Rev Neurother. 2017;17:819-837.

14. Haršány M, Tsivgoulis G, Alexandrov AV. Intravenous thrombolysis in acute ischemic stroke: standard and potential future applications. Expert Rev Neurother. 2014;14:879892.

15. Fagan SC, Morgenstern LB, Petitta A, Ward RE, Tilley $\mathrm{BC}$, Marler JR, et al. Cost-effectiveness of tissue plasminogen activator for acute ischemic stroke. NINDS rt-PA stroke study group. Neurology. 1998;50:883-890.

16. Tsivgoulis G, Goyal N, Katsanos AH, Malhotra K, Ishfaq MF, Pandhi A, et al. Intravenous thrombolysis for large vessel or distal occlusions presenting with mild stroke severi- ty. Eur J Neurol. 2020;27:1039-1047.

17. Katsanos AH, Tsivgoulis G. Is intravenous thrombolysis still necessary in patients who undergo mechanical thrombectomy? Curr Opin Neurol. 2019;32:3-12.

18. Fischer U, Kaesmacher J, Molina CA, Selim MH, Alexandrov AV, Tsivgoulis G. Primary thrombectomy in tPA (tissue-type plasminogen activator) eligible stroke patients with proximal intracranial occlusions. Stroke. 2018;49:265-269.

19. Katsanos AH, Malhotra K, Goyal N, Arthur A, Schellinger PD, Köhrmann M, et al. Intravenous thrombolysis prior to mechanical thrombectomy in large vessel occlusions. Ann Neurol. 2019;86:395-406.

20. Yang P, Treurniet KM, Zhang L, Zhang Y, Li Z, Xing P, et al. Direct intra-arterial thrombectomy in order to revascularize AIS patients with large vessel occlusion efficiently in Chinese tertiary hospitals: a multicenter randomized clinical trial (DIRECT-MT)-protocol. Int J Stroke. 2019 Oct 30. doi: 10.1177/1747493019882837 [Epub ahead of print].

21. Wechsler LR. Intravenous thrombolytic therapy for acute ischemic stroke. N Engl J Med. 2011;364:2138-2146.

22. Riedel CH, Zimmermann P, Jensen-Kondering U, Stingele R, Deuschl G, Jansen O. The importance of size: successful recanalization by intravenous thrombolysis in acute anterior stroke depends on thrombus length. Stroke. 2011;42:17751777 .

23. Tsivgoulis G, Safouris A, Krogias C, Arthur AS, Alexandrov AV. Endovascular reperfusion therapies for acute ischemic stroke: dissecting the evidence. Expert Rev Neurother. 2016;16:527-534.

24. Goyal M, Menon BK, van Zwam WH, Dippel DW, Mitchell PJ, Demchuk AM, et al. Endovascular thrombectomy after large-vessel ischaemic stroke: a meta-analysis of individual patient data from five randomised trials. Lancet. 2016;387:1723-1731.

25. Katsanos AH, Malhotra K, Goyal N, Palaiodimou L, Schellinger PD, Caso V, et al. Mortality risk in acute ischemic stroke patients with large vessel occlusion treated with mechanical thrombectomy. J Am Heart Assoc. 2019;8:e014425.

26. Aguiar de Sousa D, von Martial R, Abilleira S, Gattringer T, Kobayashi A, Gallofré M, et al. Access to and delivery of acute ischaemic stroke treatments: a survey of national scientific societies and stroke experts in 44 European countries. Eur Stroke J. 2019;4:13-28.

27. Albers GW, Molina CA, Selim MH, Jovin TG. Advanced brain imaging in late-arriving drip and ship patients with known large vessel occlusion. Stroke. 2019;50:1940-1943. 
28. Albers GW. Use of imaging to select patients for late window endovascular therapy. Stroke. 2018;49:2256-2260.

29. Demeestere J, Wouters A, Christensen S, Lemmens R, Lansberg MG. Review of perfusion imaging in acute ischemic stroke: from time to tissue. Stroke. 2020;51:1017-1024.

30. Zerna C, Thomalla G, Campbell BCV, Rha JH, Hill MD. Current practice and future directions in the diagnosis and acute treatment of ischaemic stroke. Lancet. 2018;392:1247-1256.

31. Alexandrov AV, Sloan MA, Tegeler CH, Newell DN, Lumsden A, Garami Z, et al. Practice standards for transcranial Doppler (TCD) ultrasound. Part II. Clinical indications and expected outcomes. J Neuroimaging. 2012;22:215-224.

32. Tsivgoulis G, Alexandrov AV, Sloan MA. Advances in transcranial Doppler ultrasonography. Curr Neurol Neurosci Rep. 2009;9:46-54.

33. Fischer U, Arnold M, Nedeltchev K, Brekenfeld C, Ballinari $\mathrm{P}$, Remonda L, et al. NIHSS score and arteriographic findings in acute ischemic stroke. Stroke. 2005;36:2121-2125.

34. Tsivgoulis G, Sharma VK, Lao AY, Malkoff MD, Alexandrov AV. Validation of transcranial Doppler with computed tomography angiography in acute cerebral ischemia. Stroke. 2007;38:1245-1259.

35. Campbell BCV, Mitchell PJ, Churilov L, Yassi N, Kleinig TJ, Dowling RJ, et al. Effect of Intravenous tenecteplase dose on cerebral reperfusion before thrombectomy in patients with large vessel occlusion ischemic stroke: the EXTEND-IA TNK part 2 randomized clinical trial. JAMA. 2020;323:1257-1265.

36. Tsivgoulis G, Saqqur M, Sharma VK, Brunser A, Eggers J, Mikulik R, et al. Timing of recanalization and functional recovery in acute ischemic stroke. J Stroke. 2020;22:130-140.

37. Tsivgoulis G, Sharma VK, Hoover SL, Lao AY, Ardelt AA, Malkoff MD, et al. Applications and advantages of power motion-mode Doppler in acute posterior circulation cerebral ischemia. Stroke. 2008;39:1197-1204.

38. Liberman AL, Prabhakaran S. Stroke chameleons and stroke mimics in the emergency department. Curr Neurol Neurosci Rep. 2017;17:15.

39. Tsivgoulis G, Vadikolias K, Heliopoulos I, Patousi A, Iordanidis A, Souftas V, et al. Aortic arch dissection causing acute cerebral ischemia: an uncommon contraindication for intravenous thrombolysis. Circulation. 2011;124:657-658.

40. Baumgartner RW, Mattle HP, Kothbauer K, Schroth G. Transcranial color-coded duplex sonography in cerebral aneurysms. Stroke. 1994;25:2429-2434.
41. Mastantuono JM, Combescure C, Elia N, Tramèr MR, Lysakowski C. Transcranial Doppler in the diagnosis of cerebral vasospasm: an updated meta-analysis. Crit Care Med. 2018;46:1665-1672.

42. Lepur D, Barsić B. Incidence of neurological complications in patients with native-valve infective endocarditis and cerebral microembolism: an open cohort study. Scand J Infect Dis. 2009;41:708-713.

43. Gerriets T, Goertler M, Stolz E, Postert T, Sliwka U, Schlachetzki F, et al. Feasibility and validity of transcranial duplex sonography in patients with acute stroke. J Neurol Neurosurg Psychiatry. 2002;73:17-20.

44. Herzberg M, Boy S, Hölscher T, Ertl M, Zimmermann M, Ittner KP, et al. Prehospital stroke diagnostics based on neurological examination and transcranial ultrasound. Crit Ultrasound J. 2014;6:3.

45. Hölscher T, Dunford JV, Schlachetzki F, Boy S, Hemmen T, Meyer BC, et al. Prehospital stroke diagnosis and treatment in ambulances and helicopters-a concept paper. Am J Emerg Med. 2013;31:743-747.

46. Eyding J, Fung C, Niesen WD, Krogias C. Twenty years of cerebral ultrasound perfusion imaging-is the best yet to come? J Clin Med. 2020;9:816.

47. Alexandrov AV, Burgin WS, Demchuk AM, El-Mitwalli A, Grotta JC. Speed of intracranial clot lysis with intravenous tissue plasminogen activator therapy: sonographic classification and short-term improvement. Circulation. 2001;103:2897-2902.

48. Demchuk AM, Burgin WS, Christou I, Felberg RA, Barber PA, Hill MD, et al. Thrombolysis in brain ischemia (TIBI) transcranial Doppler flow grades predict clinical severity, early recovery, and mortality in patients treated with intravenous tissue plasminogen activator. Stroke. 2001;32:89-93.

49. Tsivgoulis G, Alexandrov AV. Ultrasound in neurology. Continuum (Minneap Minn). 2016;22:1655-1677.

50. Mazya MV, Ahmed N, Azevedo E, Davalos A, Dorado L, Karlinski $\mathrm{M}$, et al. Impact of transcranial Doppler ultrasound on logistics and outcomes in stroke thrombolysis: results from the SITS-ISTR. Stroke. 2018;49:1695-1700.

51. Rha JH, Saver JL. The impact of recanalization on ischemic stroke outcome: a meta-analysis. Stroke. 2007;38:967-793.

52. Nogueira RC, Bor-Seng-Shu E, Saeed NP, Teixeira MJ, Panerai RB, Robinson TG. Meta-analysis of vascular imaging features to predict outcome following intravenous rtPA for acute ischemic stroke. Front Neurol. 2016;7:77.

53. Saqqur M, Tsivgoulis G, Molina CA, Demchuk AM, Sid- 
diqui M, Alvarez-Sabín J, et al. Symptomatic intracerebral hemorrhage and recanalization after IV rt-PA: a multicenter study. Neurology. 2008;71:1304-1312.

54. Saqqur M, Tsivgoulis G, Molina CA, Demchuk AM, Shuaib A, Alexandrov AV. Residual flow at the site of intracranial occlusion on transcranial Doppler predicts response to intravenous thrombolysis: a multi-center study. Cerebrovasc Dis. 2009;27:5-12.

55. Alexandrov AV, Tsivgoulis G, Rubiera M, Vadikolias K, Stamboulis E, Molina CA, et al. End-diastolic velocity increase predicts recanalization and neurological improvement in patients with ischemic stroke with proximal arterial occlusions receiving reperfusion therapies. Stroke. 2010;41:948-952.

56. Alexandrov AV, Demchuk AM, Felberg RA, Grotta JC, Krieger DW. Intracranial clot dissolution is associated with embolic signals on transcranial Doppler. J Neuroimaging. 2000;10:27-32.

57. Tsivgoulis G, Alexandrov A. Ultrasound-enhanced thrombolysis: from bedside to bench. Stroke. 2008;39:1404-1405.

58. Bang OY, Saver JL, Kim SJ, Kim GM, Chung CS, Ovbiagele $\mathrm{B}$, et al. Collateral flow predicts response to endovascular therapy for acute ischemic stroke. Stroke. 2011;42:693-699.

59. Saqqur M, Uchino K, Demchuk AM, Molina CA, Garami $Z$, Calleja S, et al. Site of arterial occlusion identified by transcranial Doppler predicts the response to intravenous thrombolysis for stroke. Stroke. 2007;38:948-954.

6o. Tsivgoulis G, Ribo M, Rubiera M, Vasdekis SN, Barlinn $\mathrm{K}$, Athanasiadis D, et al. Real-time validation of transcranial Doppler criteria in assessing recanalization during intra-arterial procedures for acute ischemic stroke: an international, multicenter study. Stroke. 2013;44:394-400.

61. Sekoranja L, Loulidi J, Yilmaz H, Lovblad K, Temperli $\mathrm{P}$, Comelli $\mathrm{M}$, et al. Intravenous versus combined (intravenous and intra-arterial) thrombolysis in acute ischemic stroke: a transcranial color-coded duplex sonography--guided pilot study. Stroke. 2006;37:1805-1809.

62. Rubiera M, Cava L, Tsivgoulis G, Patterson DE, Zhao L, Zhang Y, et al. Diagnostic criteria and yield of real-time transcranial Doppler monitoring of intra-arterial reperfusion procedures. Stroke. 2010;41:695-699.

63. Effects of tissue plasminogen activator and a comparison of early invasive and conservative strategies in unstable angina and non-q-wave myocardial infarction. Results of the TIMI IIIb trial. Thrombolysis in myocardial ischemia. Circulation. 1994;89:1545-1556.
64. Baracchini C, Farina F, Palmieri A, Kulyk C, Pieroni A, Viaro F, et al. Early hemodynamic predictors of good outcome and reperfusion injury after endovascular treatment. Neurology. 2019;92:e2774-e2783.

65. Seners P, Turc G, Oppenheim C, Baron JC. Incidence, causes and predictors of neurological deterioration occurring within $24 \mathrm{~h}$ following acute ischaemic stroke: a systematic review with pathophysiological implications. J Neurol Neurosurg Psychiatry. 2015;86:87-94.

66. Tisserand M, Seners P, Turc G, Legrand L, Labeyrie MA, Charron S, et al. Mechanisms of unexplained neurological deterioration after intravenous thrombolysis. Stroke. 2014;45:3527-3534.

67. Seners P, Hurford R, Tisserand M, Turc G, Legrand L, Naggara $\mathrm{O}$, et al. Is unexplained early neurological deterioration after intravenous thrombolysis associated with thrombus extension? Stroke. 2017;48:348-352.

68. Kim JM, Bae JH, Park KY, Lee WJ, Byun JS, Ahn SW, et al. Incidence and mechanism of early neurological deterioration after endovascular thrombectomy. J Neurol. 2019;266:609-615.

69. Pérez ES, Delgado-Mederos R, Rubiera M, Delgado P, Ribó $\mathrm{M}$, Maisterra $\mathrm{O}$, et al. Transcranial duplex sonography for monitoring hyperacute intracerebral hemorrhage. Stroke. 2009;40:987-990.

70. Seidel G, Cangür H, Albers T, Meyer-Wiethe K. Transcranial sonographic monitoring of hemorrhagic transformation in patients with acute middle cerebral artery infarction. J Neuroimaging. 2005;15:326-330.

71. Seidel G, Cangür H, Albers T, Burgemeister A, Meyer-Wiethe K. Sonographic evaluation of hemorrhagic transformation and arterial recanalization in acute hemispheric ischemic stroke. Stroke. 2009;40:119-123.

72. Alexandrov AV, Grotta JC. Arterial reocclusion in stroke patients treated with intravenous tissue plasminogen activator. Neurology. 2002;59:862-867.

73. Rubiera M, Alvarez-Sabín J, Ribo M, Montaner J, Santamarina E, Arenillas JF, et al. Predictors of early arterial reocclusion after tissue plasminogen activator-induced recanalization in acute ischemic stroke. Stroke. 2005;36:1452-1456.

74. Li W, Ding J, Sui X, Qi Z, Wu L, Sun C, et al. Prognosis and risk factors for reocclusion after mechanical thrombectomy. Ann Clin Transl Neurol. 2020;7:420-428.

75. Marto JP, Strambo D, Hajdu SD, Eskandari A, Nannoni $S$, Sirimarco G, et al. Twenty-four-hour reocclusion after successful mechanical thrombectomy: associated factors 
and long-term prognosis. Stroke. 2019;50:2960-2963.

76. Mosimann PJ, Kaesmacher J, Gautschi D, Bellwald S, Panos L, Piechowiak E, et al. Predictors of unexpected early reocclusion after successful mechanical thrombectomy in acute ischemic stroke patients. Stroke. 2018;49:2643-2651.

77. Tsivgoulis G, Eggers J, Ribo M, Perren F, Saqqur M, Rubiera $\mathrm{M}$, et al. Safety and efficacy of ultrasound-enhanced thrombolysis: a comprehensive review and meta-analysis of randomized and nonrandomized studies. Stroke. 2010;41:280287.

78. Molina CA, Alexandrov AV, Demchuk AM, Saqqur M, Uchino K, Alvarez-Sabín J, et al. Improving the predictive accuracy of recanalization on stroke outcome in patients treated with tissue plasminogen activator. Stroke. 2004;35:151-156.

79. Palazzo P, Balucani C, Barlinn K, Tsivgoulis G, Zhang Y, Zhao L, et al. Association of reversed Robin Hood syndrome with risk of stroke recurrence. Neurology. 2010;75:2003-2008.

8o. Alexandrov AV, Nguyen HT, Rubiera M, Alexandrov AW, Zhao L, Heliopoulos I, et al. Prevalence and risk factors associated with reversed Robin Hood syndrome in acute ischemic stroke. Stroke. 2009;40:2738-2742.

81. Tsivgoulis G, Saqqur M, Sharma VK, Lao AY, Hill MD, Alexandrov AV; CLOTBUST Investigators. Association of pretreatment blood pressure with tissue plasminogen activator-induced arterial recanalization in acute ischemic stroke. Stroke. 2007;38:961-966.

82. Molina CA, Alvarez-Sabín J, Montaner J, Abilleira S, Arenillas JF, Coscojuela P, et al. Thrombolysis-related hemorrhagic infarction: a marker of early reperfusion, reduced infarct size, and improved outcome in patients with proximal middle cerebral artery occlusion. Stroke. 2002;33:1551-1556.

83. Saqqur M, Shuaib A, Alexandrov AV, Hill MD, Calleja $\mathrm{S}$, Tomsick T, et al. Derivation of transcranial Doppler criteria for rescue intra-arterial thrombolysis: multicenter experience from the Interventional Management of Stroke study. Stroke. 2005;36:865-868.

84. Sheriff F, Diz-Lopes M, Khawaja A, Sorond F, Tan CO, Azevedo $\mathrm{E}$, et al. Microemboli after successful thrombectomy do not affect outcome but predict new embolic events. Stroke. 2020;51:154-161.

85. Kargiotis O, Psychogios K, Safouris A, Magoufis G, Zervas PD, Stamboulis E, et al. The role of transcranial Doppler monitoring in patients with multi-territory acute embolic strokes: a review. J Neuroimaging. 2019;29:309-322.

86. Paciaroni M, Balucani C, Agnelli G, Caso V, Silvestrelli G, Grotta JC, et al. Systemic thrombolysis in patients with acute ischemic stroke and Internal Carotid ARtery Occlusion: the ICARO study. Stroke. 2012;43:125-130.

87. Cardim D, Robba C, Bohdanowicz M, Donnelly J, Cabella B, Liu X, et al. Non-invasive monitoring of intracranial pressure using transcranial Doppler ultrasonography: is it possible? Neurocrit Care. 2016;25:473-491.

88. Motuel J, Biette I, Srairi M, Mrozek S, Kurrek MM, Chaynes $\mathrm{P}$, et al. Assessment of brain midline shift using sonography in neurosurgical ICU patients. Crit Care. 2014;18:676.

89. Chen H, Su Y, He Y, Zhang Y, Sun Y, Fan L, et al. Controlling blood pressure under transcranial doppler guidance after endovascular treatment in patients with acute ischemic stroke. Cerebrovasc Dis. 2020;49:160-169. 